\title{
Dopamine-Mediated Zwitterionic Polyelectrolyte Coated Polypropylene Hernia Mesh with Synergistic Anti-Inflammation Effects
}

Yansha Qiao, Yan Li*, Qian Zhang, Qian Wang, Jing Gao, and Lu Wang* Key Laboratory of Textile Science \&Technology, Ministry of Education, College of Textiles, Donghua University, Shanghai 201620, China

* Corresponding author

Dr. Yan Li or Prof. Lu Wang

Key Laboratory of Textile Science \&Technology, Ministry of Education, College of Textiles, Donghua University, Shanghai 201620, China.

Tel: +86-21-67792634 (Y. LI). Tel: +86-21-67792637; Fax: +86-21-67792637. (L. WANG)

E-mail addresses: yanli@dhu.edu.cn (Y. LI) or wanglu@dhu.edu.cn (L. WANG) 


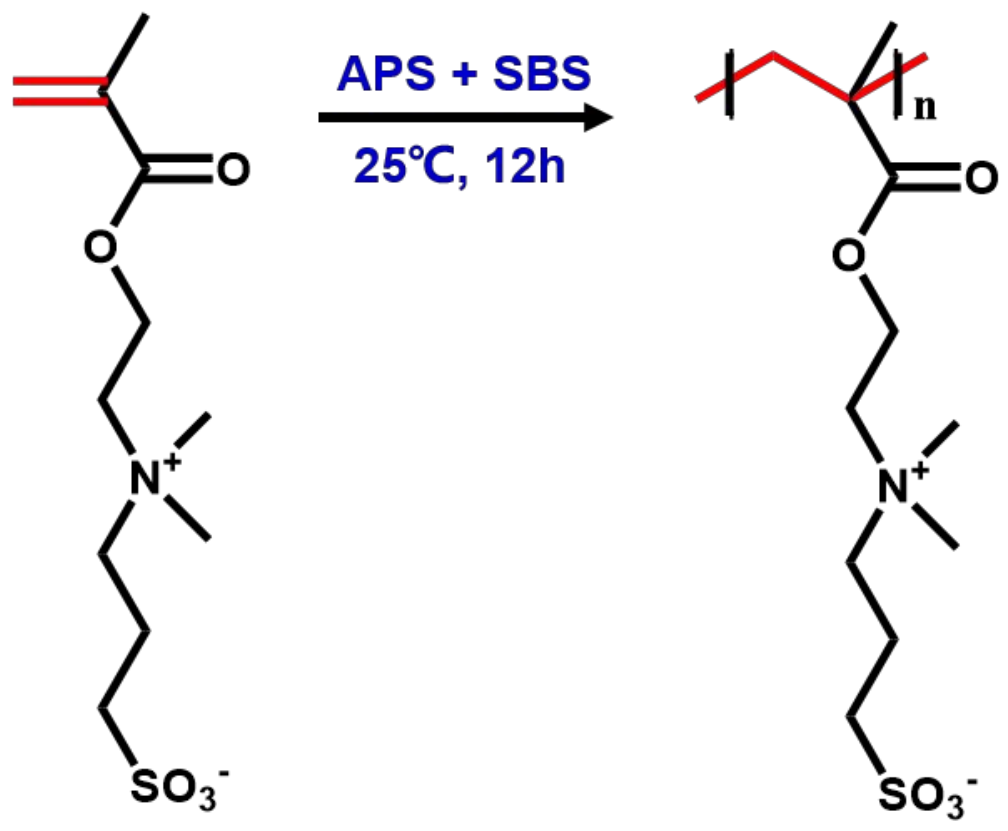

Scheme S1. Synthetic route of PSBMA.

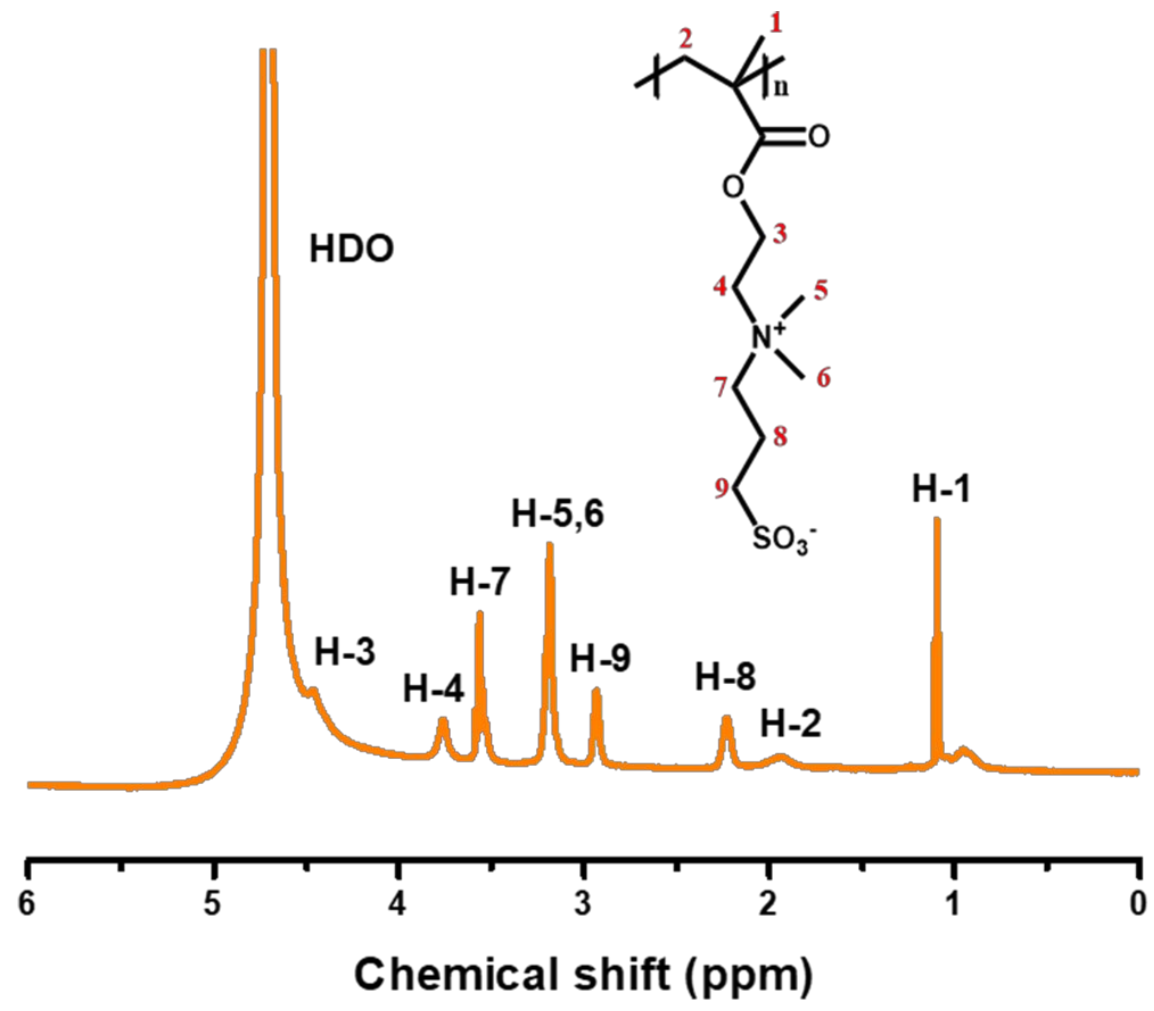

Figure S1. ${ }^{1} \mathrm{H}$ NMR spectrum of PSBMA. 
(a)
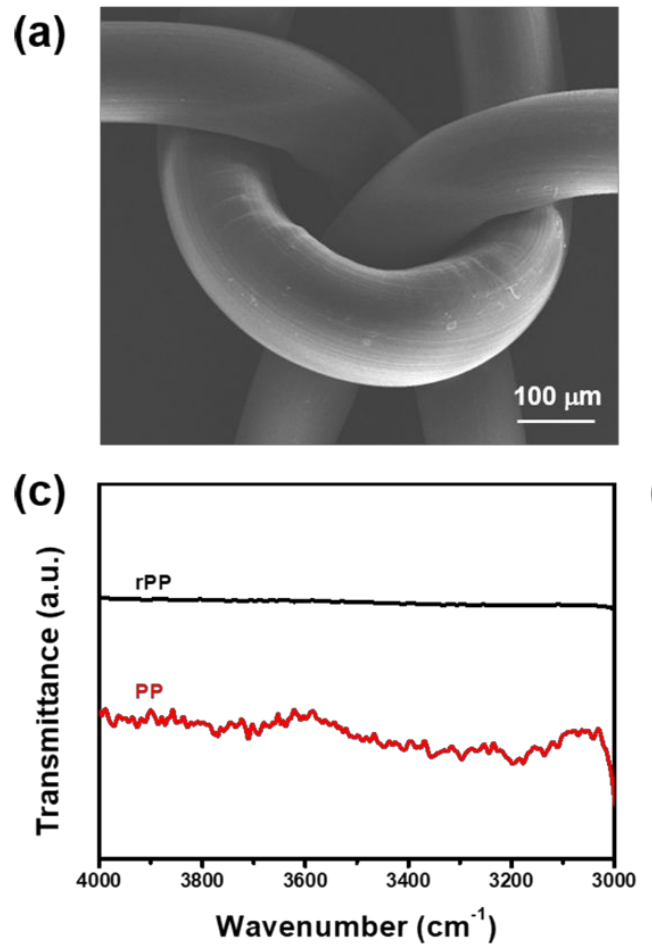

(b)

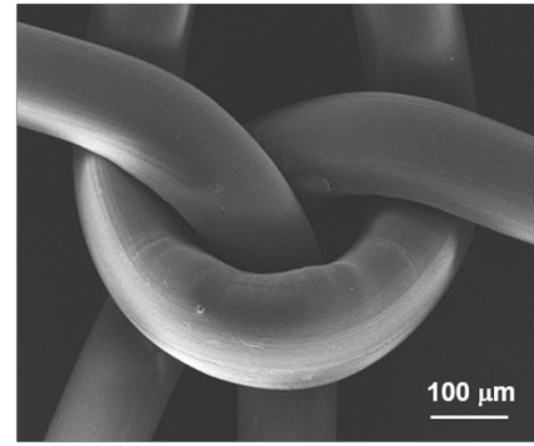

(d)

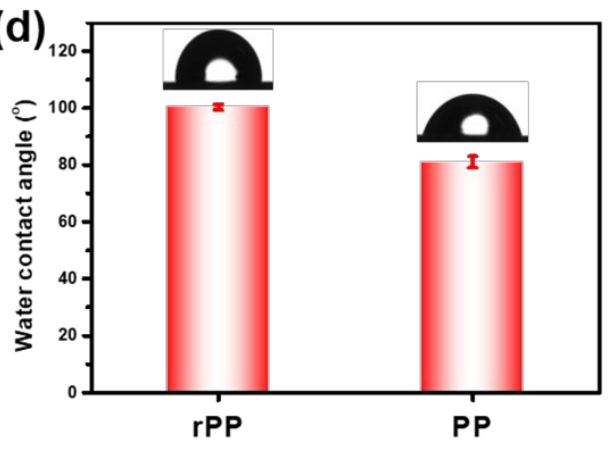

Figure S2. The SEM images of (a) rPP and (b) PP monofilaments, (c) ATR-FTIR spectra and (d) WCA of rPP and PP

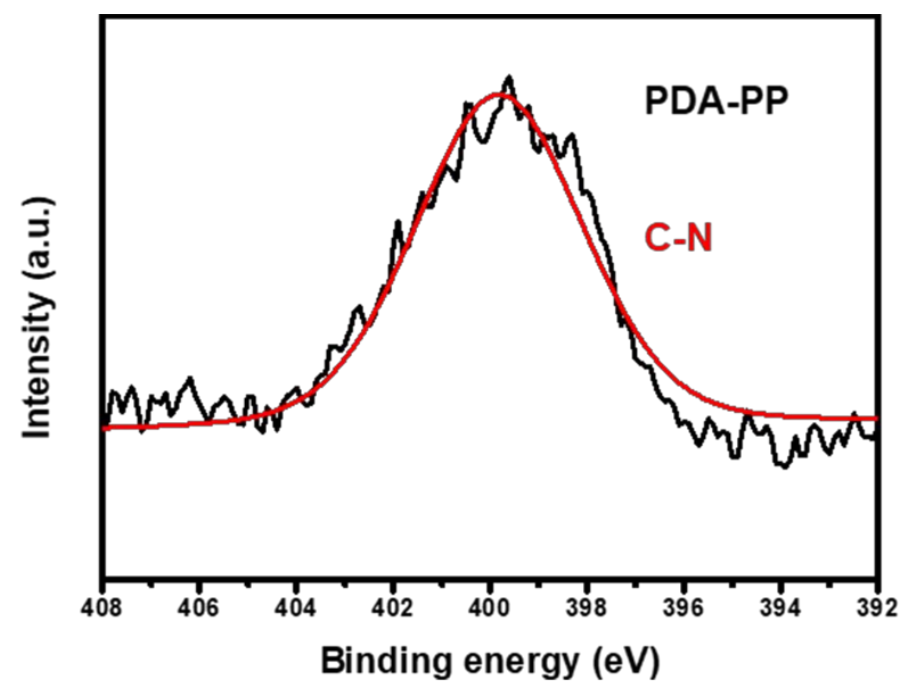

Figure S3. The high-resolution scans of N1s of PDA-PP. 

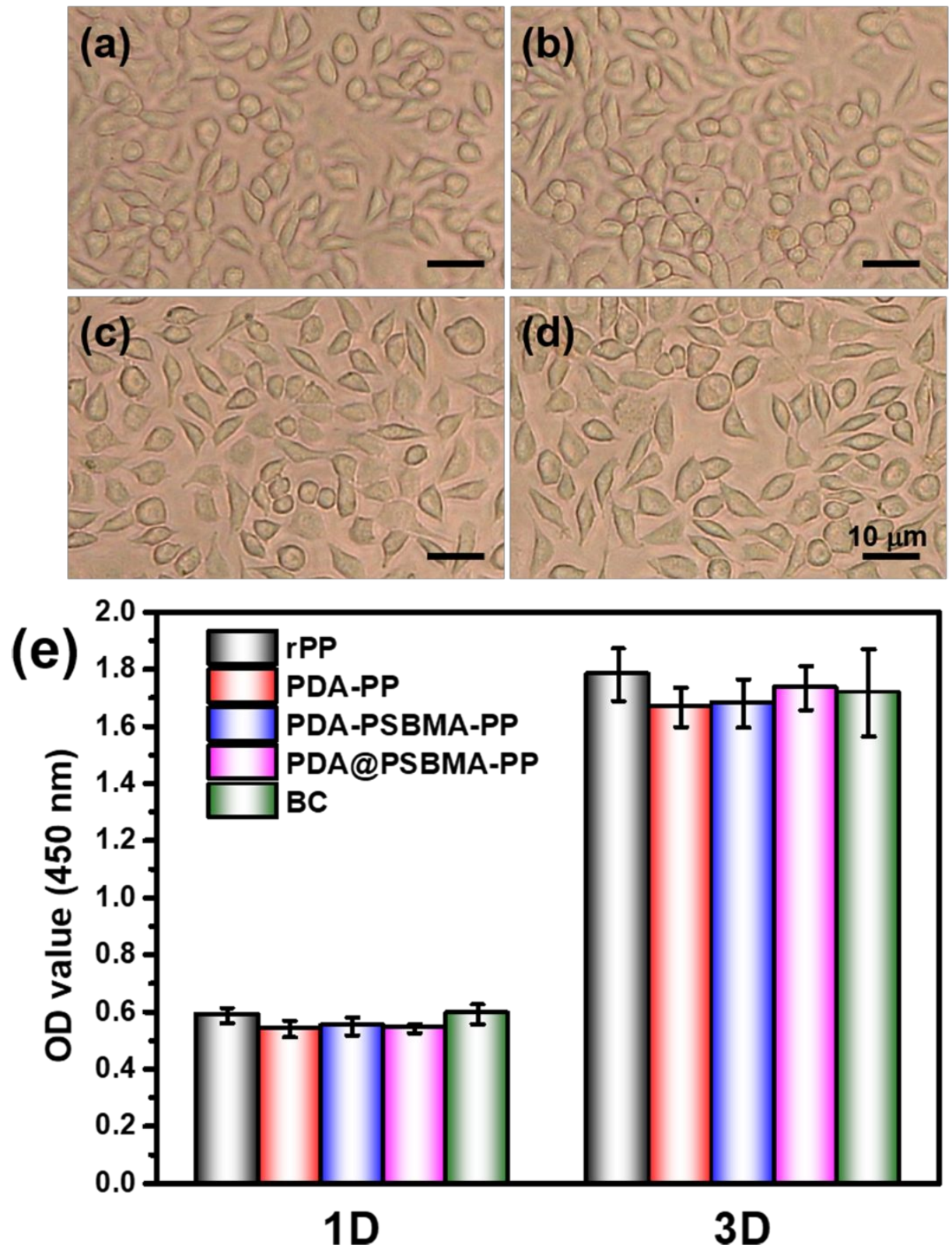

Figure S4. Optical microscope images of L929 cells incubated in medium soaked with rPP (a), PDA-PP (b), PSBMA-PDA-PP (c), PSBMA@PDA-PP (d). The OD values of cytotoxicity test results from CCK-8. 
Table S1. The surface elemental composition of PSBMA-PDA-PP

\begin{tabular}{ccccc}
\hline $\begin{array}{c}\text { Concentrations of } \\
\text { PSBMA (mg/mL) }\end{array}$ & $\mathbf{C}(\%)$ & $\mathbf{N}(\%)$ & $\mathbf{O}(\%)$ & $\mathbf{S ~ ( \% )}$ \\
\hline 2 & 68.92 & 7.04 & 22.52 & 1.52 \\
\hline 0.4 & 69.37 & 7.67 & 21.96 & 1.00 \\
\hline 0.2 & 69.52 & 7.13 & 22.46 & 0.89 \\
\hline
\end{tabular}

Table S2. The surface elemental composition of rPP and PP

\begin{tabular}{ccc}
\hline Mesh & C (\%) & O (\%) \\
\hline rPP & 100 & 0 \\
\hline PP & 88.18 & 11.82 \\
\hline
\end{tabular}

Table S3. The surface elemental composition of PDA-PP and PSBMA@PDA-PP

\begin{tabular}{ccccc}
\hline Sample & $\mathbf{C ~ ( \% )}$ & $\mathbf{N}(\%)$ & $\mathbf{O}(\%)$ & $\mathbf{S ~ ( \% )}$ \\
\hline PDA-PP & 65.61 & 6.76 & 27.63 & 0 \\
\hline PSBMA@PDA-PP & 71.59 & 7.47 & 19.83 & 1.10 \\
\hline
\end{tabular}

Table S4. The surface elemental composition of degraded samples on 7 D

\begin{tabular}{ccccc}
\hline Sample & $\mathbf{C ~ ( \% )}$ & $\mathbf{N}(\%)$ & $\mathbf{O}(\boldsymbol{\%})$ & $\mathbf{S ~ ( \% )}$ \\
\hline PSBMA-PDA-PP & 67.38 & 7.10 & 24.78 & 0.74 \\
\hline PSBMA@PDA-PP & 69.06 & 6.92 & 23.20 & 0.82 \\
\hline
\end{tabular}

